\title{
Coupled Harmonic Equations, SOR, and Chebyshev Acceleration*
}

\author{
By L. W. Ehrlich
}

\begin{abstract}
A coupled pair of harmonic equations is solved by the application of Chebyshev acceleration to the Jacobi, Gauss-Seidel, and related iterative methods, where the Jacobi iteration matrix has purely imaginary (or zero) eigenvalues. Comparison is made with a block SOR method used to solve the same problem.
\end{abstract}

Introduction. In [4], we proposed a general block SOR method for solving the biharmonic equation as a coupled set of finite-difference equations. Here, we consider related methods and compare them to the SOR method. The methods considered here are Chebyshev accelerated Jacobi and Gauss-Seidel as well as others, the cyclic Chebyshev semi-iteration method [5], and the unsymmetric modified SOR method [20], [21]. It is shown, by comparing spectral radii, that the SOR method of [4] is at least as fast as any of the above methods. The analysis applies to a block cyclic matrix of index 2 , or one that can be written in the form (2.1). The interesting feature of the analysis is that the Jacobi matrix has purely imaginary (or zero) eigenvalues, whereas most previous work assumes real eigenvalues, or some complex.

To fix notation, consider the iterative process

$$
u^{(n+1)}=G u^{(n)}+d,
$$

where there exists a vector $u$ such that

$$
u=G u+d .
$$

Let $\lambda_{i}(G)$ be an eigenvalue of $G$ and let $S(G)=\bar{\lambda}=\max _{i}\left|\lambda_{i}(G)\right|$ be the spectral radius of $G$. The rate of convergence of the iterative process $(0.1)$ is defined by

$$
R(G)=-\ln S(G)=-\ln \bar{\lambda},
$$

when $\bar{\lambda}<1[18]$. In the event that $G$ is a function of $n$, i.e.,

$$
u^{(n+1)}=G_{n} u^{(n)}+d,
$$

we define the average rate of convergence as [16]

$$
R\left(G_{n}\right)=-\left(\ln S\left(G_{n}\right)\right) / n
$$

Received April 27, 1971, revised October 14, 1971.

AMS 1970 subject classifications. Primary 65F10, 65N20, 15A06.

Key words and phrases. Chebyshev acceleration, successive overrelaxation, biharmonic equation, coupled harmonic equations, harmonic equations, finite differences, iterative solutions of linear systems.

* This work was supported by the Department of the Navy, Naval Ordnance Systems Command, under Contract N00017-62-C-0604. 
and the asymptotic average rate of convergence as [16]

$$
R_{\infty}\left(G_{n}\right)=\lim _{n \rightarrow \infty}-\frac{\ln S\left(G_{n}\right)}{n}
$$

1. The Matrix Equation. Consider

$$
\begin{aligned}
\Delta^{2} u \equiv u_{x x x x}+2 u_{x x y y}+u_{y y y}=0, & 0<x<1,0<y<1, \\
u & =0, \quad x=0,1 \text { or } y=0,1, \\
u_{n} & =0, \quad x=0,1 \text { or } y=0, \\
u_{n} & =1, \quad y=1,
\end{aligned}
$$

where $u_{n}$ is the outward normal derivative on the boundary of the unit square. The Eq. (1.1) can be replaced by

$$
\begin{aligned}
\Delta u & \equiv u_{x x}+u_{y y}=v, \quad 0<x, y<1, \\
\Delta v & \equiv v_{x x}+v_{y y}=0,
\end{aligned}
$$

with boundary conditions (1.2).

As in [4], [13], superimpose a square grid over the unit square with mesh size $h=1 /(N+1)$, for some positive integer $N$. Approximating $\Delta u$ and the normal derivatives in the usual manner, we are led to the matrix system

$$
\begin{aligned}
L u-h^{2} v & =0, \\
L v+2 M u / h^{2} & =D / h^{2}
\end{aligned}
$$

or

$$
\left(L^{2}+2 M\right) u=D,
$$

where $D$ contains the boundary conditions and where

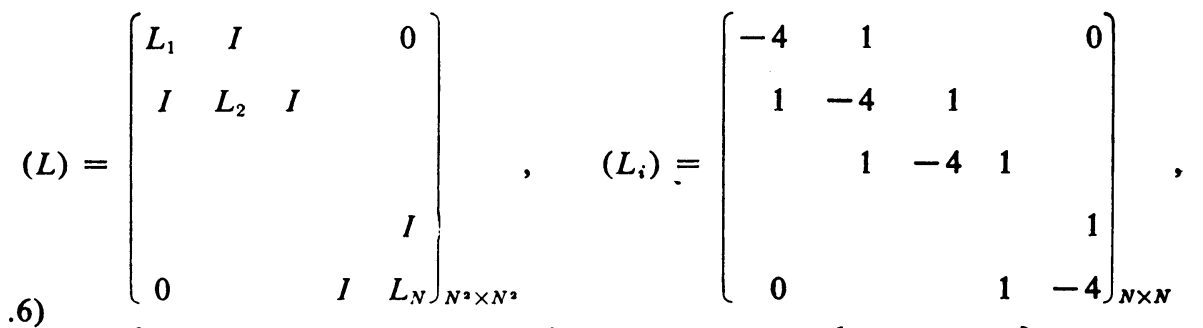

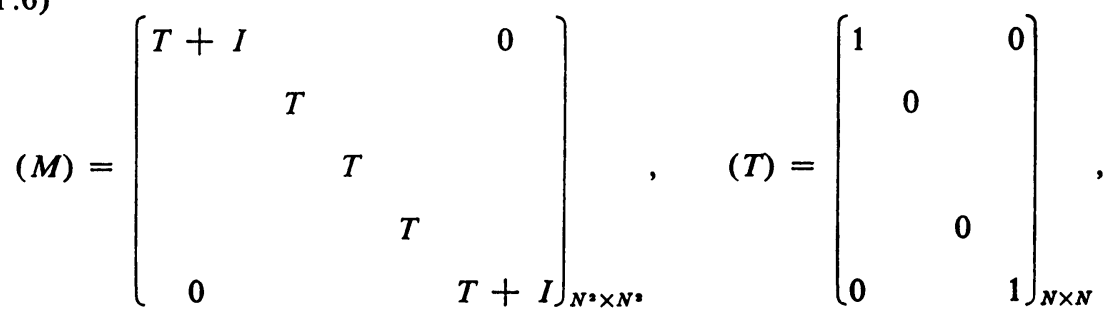

where $I$ is the $N \times N$ identity (see [4], [13] for details). 
2. The Modified (Block) SOR Method. Equation (1.4) can be written in the partitioned matrix form

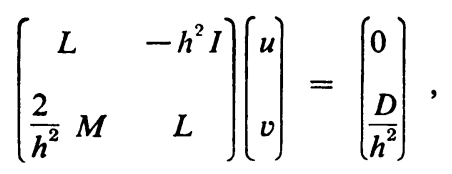

where $L^{-1}$ exists. We may consider this a linear system in a block cyclic form of index 2 [16] or a block $\sigma_{1}$ ordering form [18], [21]. The Modified Successive Overelaxation Method (MSOR) [20], [21], [22], [23] applied to (2.1) is

$$
\begin{aligned}
& u^{(n+1)}=\omega_{1}\left(h^{2} L^{-1} v^{(n)}\right)+\left(1-\omega_{1}\right) u^{(n)}, \\
& v^{(n+1)}=\omega_{2}\left(-\frac{2}{h^{2}} L^{-1} M u^{(n+1)}+\frac{L^{-1} D}{h^{2}}\right)+\left(1-\omega_{2}\right) v^{(n)},
\end{aligned}
$$

or

$$
\boldsymbol{w}^{(n+1)}=\mathfrak{R}_{\omega_{1}, \omega_{2}} \boldsymbol{w}^{(n)}+k,
$$

where

$$
w^{(n)}=\left(\begin{array}{l}
u^{(n)} \\
v^{(n)}
\end{array}\right),
$$

$$
\begin{gathered}
\mathfrak{R}_{\omega_{1, \omega_{2}}}=\left(\begin{array}{cc}
\left(1-\omega_{1}\right) I & \omega_{1} h^{2} L^{-1} \\
-\frac{2 \omega_{2}}{h^{2}}\left(1-\omega_{1}\right) L^{-1} M & \left(1-\omega_{2}\right) I-2 \omega_{1} \omega_{2} L^{-1} M L^{-1}
\end{array}\right), \\
k=\left(\begin{array}{c}
0 \\
\omega_{2} L^{-1} D / h^{2}
\end{array}\right) .
\end{gathered}
$$

This is precisely the iterative method considered in [4] and analyzed there. However, the basis of this analysis appears also in [21], [22].

Let

$$
B=\left(\begin{array}{cc}
0 & h^{2} L^{-1} \\
-\frac{2}{h^{2}} L^{-1} M & 0
\end{array}\right) .
$$

This is the Jacobi iteration matrix for the linear system (2.1). If $\mu$ is an eigenvalue of $B$, then $\lambda$ is an eigenvalue of $\mathfrak{R}_{\omega_{1}, \omega_{2}}$, where [21], [22]

$$
\left(\lambda+\omega_{1}-1\right)\left(\lambda+\omega_{2}-1\right)=\omega_{1} \omega_{2} \lambda \mu^{2} .
$$

To find an expression for $\mu$, let $\left(\begin{array}{c}x_{1} \\ x_{3}\end{array}\right)$ be a partitioned eigenvector of $B$. Then

$$
h^{2} L^{-1} x_{2}=\mu x_{1}, \quad-\frac{2}{h^{2}} L^{-1} M x_{1}=\mu x_{2},
$$

and, eliminating $\boldsymbol{x}_{2}$, we have

$$
2 L^{-2} M x_{1}=-\mu^{2} x_{1}
$$


If $\tau$ is an eigenvalue of $L^{-2} M$, where $0 \leqq \tau \leqq \bar{\tau}=S\left(L^{-2} M\right)$, then

$$
\mu^{2}=-2 \tau
$$

and all the eigenvalues of $B$ are purely imaginary (or zero). Equation (2.8) becomes

$$
\left(\lambda+\omega_{1}-1\right)\left(\lambda+\omega_{2}-1\right)=-2 \omega_{1} \omega_{2} \lambda \tau
$$

which is Eq. (3.6) of [4]. The optimal $\omega$ 's with regard to $S\left(R_{\omega_{1}, \omega_{2}}\right)$ occur when $\omega_{1}=\omega_{2}$, where (2.12) becomes

$$
(\lambda+\omega-1)^{2}=-2 \omega^{2} \lambda \tau .
$$

We shall call this the SOR method. The optimal $\omega$ has been determined as a function $\mu$ in [21], [22] (see also [8]) and we have

$$
\omega_{0}=\frac{2}{1+(1+2 \bar{\tau})^{1 / 2}},
$$

where $\bar{\tau}=S\left(L^{-2} M\right)$, and where the matrix is denoted as $\mathfrak{l}_{\omega_{0}}=\mathfrak{l}_{\omega_{0}, \omega_{0}}$. Then,

$$
S\left(\mathfrak{Q}_{\omega_{0}}\right)=\bar{\lambda}=1-\omega_{0}=\frac{(1+2 \bar{\tau})^{1 / 2}-1}{(1+2 \bar{\tau})^{1 / 2}+1}=\frac{2 \bar{\tau}}{\left(1+(1+2 \bar{\tau})^{1 / 2}\right)^{2}} .
$$

Furthermore, using Lemma 6-2.1 of [21], we have that, for convergence (i.e., $\bar{\lambda}<1$ ), we must have

$$
2 \omega^{2} \bar{\tau}<(\omega-2)^{2}
$$

or, convergence occurs for

$$
0<\omega<\bar{\omega}=\frac{2}{1+(2 \bar{\tau})^{1 / 2}} .
$$

(These results are also available in [9], [10], [10a].)

In the Appendix of [4], we have shown that

$$
.25 / h \leqq \bar{\tau} \leqq .339 / h \text {. }
$$

Let $\bar{\tau}=c / h$ for some constant $c$. Then, for small $h$, we have

$$
\begin{aligned}
\omega_{0} & \approx 2\left(\frac{h}{2 c}\right)^{1 / 2}-2\left(\frac{h}{2 c}\right)+\left(\frac{h}{2 c}\right)^{3 / 2}+\cdots, \\
\bar{\omega} & \approx 2\left(\frac{h}{2 c}\right)^{1 / 2}-2\left(\frac{h}{2 c}\right)+2\left(\frac{h}{2 c}\right)^{3 / 2}+\cdots .
\end{aligned}
$$

Thus, the optimal $\omega$ is very near the divergent $\omega$ range (this has also been observed in [9], [11], [17], [21]).

3. Chebyshev Acceleration and Cyclic Semi-Iteration. When the basic iteration matrix is similar to a diagonal matrix and all eigenvalues are real and lie in the interval $[a, b]$, the Chebyshev acceleration scheme [16], [19] employs the Chebyshev polynomials

$$
P_{n}(x)=T_{n}(\gamma) / T_{n}(d)
$$

where 


$$
\begin{aligned}
\gamma & =\frac{2 x-(b+a)}{b-a}, & & \\
d & =\frac{2-(b+a)}{b-a}, & & \\
T_{n}(\gamma) & =\cos \left(n \cos ^{-1} \gamma\right), & & \gamma \text { real },|\gamma|<1, \\
& =\cosh \left(n \cosh ^{-1} \gamma\right), & & \text { otherwise. }
\end{aligned}
$$

If the basic iteration scheme is convergent, the Chebyshev process is an order of magnitude faster [3], [16], [19].

If some of the eigenvalues of the basic matrix are complex, the efficiency of the Chebyshev scheme is a function of the eccentricity of the ellipse which contains all the eigenvalues, and this efficiency decreases to zero as the ellipse degenerates into a circle [17]. Thus, if all eigenvalues lie on a circle, the Chebyshev process does not improve the basic method [15], [17].

Here, we consider the basic method having eigenvalues which are purely imaginary (or zero). We consider the Chebyshev scheme (3.1) with

$$
\begin{aligned}
\gamma^{\prime} & =\frac{2 i y-(i b+i a)}{i b-i a}=\frac{2 y-(b+a)}{b-a}, \\
d^{\prime} & =\frac{2-i(b+a)}{i(b-a)},
\end{aligned}
$$

where the eigenvalues are $i y$ and

$$
a \leqq y \leqq b .
$$

We shall apply this Chebyshev scheme to the Jacobi iteration matrix $B$ of (2.7). From (2.11), the eigenvalues of $B$ are of the form $i y$ where

$$
-(2 \bar{\tau})^{1 / 2} \leqq y \leqq(2 \bar{\tau})^{1 / 2} .
$$

Thus, $\gamma^{\prime}=y /(2 \bar{\tau})^{1 / 2}, d^{\prime}=1 / i(2 \bar{\tau})^{1 / 2}$ and we have

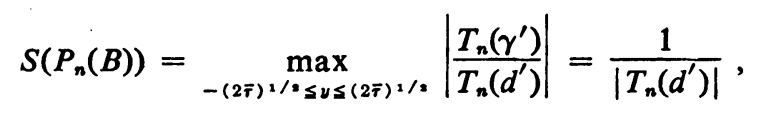

since $\gamma^{\prime}$ is real and $\left|\gamma^{\prime}\right|<1$.

From (3.2), we have

$$
S\left(P_{n}(B)\right)=\left|\frac{2\left(\omega_{0}-1\right)^{n / 2}}{1+\left(\omega_{0}-1\right)^{n}}\right|=\frac{2\left(1-\omega_{0}\right)^{n / 2}}{1+\left(\omega_{0}-1\right)^{n}} .
$$

One can show, using the results of [17], that

$$
R_{\infty}\left(P_{n}(B)\right)=\lim _{n \rightarrow \infty}-\frac{\ln \left(S\left(P_{n}(B)\right)\right)}{n}=\sinh ^{-1} \frac{1}{(2 \bar{\tau})^{1 / 2}} .
$$

(For real $d, R_{\infty}=\cosh ^{-1} d$.)

By (2.17), we see that the Jacobi method is itself not convergent. However, the Chebyshev scheme based on the Jacobi method is convergent. To compare the SOR method and the accelerated Jacobi method, we consider the ratio of $R\left(\mathfrak{Q}_{\omega_{0}}\right)$ to $R\left(P_{n}(B)\right)$. As in [15], we obtain the result 


$$
R\left(\mathfrak{R}_{\omega_{0}}\right) / R\left(P_{n}(B)\right) \geqq 2,
$$

which implies that the SOR method is at least twice as fast.

We now consider the Chebyshev scheme applied to the Gauss-Seidel iteration, i.e.,

$$
\mathfrak{R}_{1}=\left(\begin{array}{cc}
0 & h^{2} L^{-1} \\
0 & -2 L^{-1} M L^{-1}
\end{array}\right) .
$$

From (2.13), the eigenvalues of this matrix are real and lie in the interval $[-2 \bar{\tau}, 0]$. The Chebyshev scheme here is (3.1), with

$$
\begin{aligned}
& \gamma=(2 x+2 \bar{\tau}) / 2 \bar{\tau}=(x+\bar{\tau}) / \bar{\tau}, \\
& d=(1+\bar{\tau}) / \bar{\tau}
\end{aligned}
$$

From (3.2), we have

$$
S\left(P_{n}\left(\Omega_{1}\right)\right)=\frac{2\left(1-\omega_{0}\right)^{n}}{1+\left(1-\omega_{0}\right)^{2 n}} .
$$

Again, since the basic method is not convergent, we compare this with SOR and obtain, as in [15],

$$
R\left(\mathfrak{R}_{\omega_{0}}\right) / R\left(P_{n}\left(\mathfrak{R}_{1}\right)\right) \geqq 1,
$$

with equality in the limit. Thus, the SOR method is at least as fast.

We cannot improve on the SOR method itself, because at $\omega_{0}$, from (2.13), the eigenvalues lie on a circle of radius $\left(1-\omega_{0}\right)$ in the complex plane.

In [14], Smith considered the scheme (2.2) with $\omega_{2}=1$, i.e.,

$$
R_{\omega_{1,1}}=\left(\begin{array}{cc}
\left(1-\omega_{1}\right) I & \omega_{1} h^{2} L^{-1} \\
-\frac{2}{h^{2}}\left(1-\omega_{1}\right) L^{-1} M & -2 \omega_{1} L^{-1} M L^{-1}
\end{array}\right) .
$$

The eigenvalues $\lambda$ of $\mathfrak{R}_{\omega_{1,2}}$ are determined by $\lambda+\omega_{1}-1=-2 \omega_{1} \tau$ (2.12) and the optimal $\omega_{1}$ occurs when $1-\omega_{1}-2 \omega_{1} \tau=\omega_{1}-1$, since $0 \leqq \tau \leqq \bar{\tau}$. Thus, the optimal $\omega_{1}=1 /(1+\bar{\tau})$, and we have that the eigenvalues are real and lie in the interval $[-\bar{\tau} /(1+\bar{\tau}), \bar{\tau} /(1+\bar{\tau})]$. We then have $\gamma=x(1+\bar{\tau}) / \bar{\tau}, d=(1+\bar{\tau}) / \bar{\tau}$. Here, the basic method is convergent and the Chebyshev acceleration improves the convergence by an order of magnitude [14]. The spectral radius of this method is given in (3.12) and we have also

$$
\frac{R\left(R_{\omega_{0}}\right)}{R\left(P_{n}\left(R_{\omega_{1}, 1}\right)\right)} \geqq \frac{-\ln \left(2 \bar{\tau} /\left(1+(1+2 \bar{\tau})^{1 / 2}\right)^{2}\right)}{\cosh ^{-1}((1+\tau) / \tau)}=1 \text {, }
$$

indicating the SOR is again at least as fast [4], [14].

Another approach is to consider iterating the first time with $\omega=1$, followed by $\omega=\omega_{0}$ for all subsequent iterations. This was first suggested by Sheldon [12] for matrices of the form (2.1). From the analysis of [5], [21, Chapters 7 and 10], one can show that the matrix of this method has spectral radius of

$$
4\left(1-\omega_{0}\right)^{m}\left(\left(2-\omega_{0}\right) m-\left(1-\omega_{0}\right)\right) / \omega_{0}^{2}
$$

after $m$ iterations. Asymptotically, this is as fast as the SOR method. 
Finally, we consider

$$
\begin{aligned}
& u^{(2 n+1)}=\omega_{2 n+1}\left(h^{2} L^{-1} v^{(2 n)}\right)+\left(1-\omega_{2 n+1}\right) u^{(2 n-1)}, \\
& v^{(2 n+2)}=\omega_{2 n+2}\left(-\frac{2}{h^{2}} L^{-1} M u^{(2 n+1)}+\frac{L^{-1} D}{h^{2}}\right)+\left(1-\omega_{2 n+2}\right) v^{(2 n)}
\end{aligned}
$$

which is the cyclic Chebyshev semi-iteration method (CCSOR) of Golub and Varga [5]. If we let

$$
\begin{aligned}
\omega_{1} & =1, \quad \omega_{2}=1 /(1+\bar{\tau}), \\
\omega_{i+1} & =1 /\left(1+\frac{1}{2} \bar{\tau} \omega_{i}\right), \quad i \geqq 2,
\end{aligned}
$$

then again using the analysis of [5], [21, Chapter 10], we can show that the spectral radius of the above method is

$$
2\left(1-\omega_{0}\right)^{m} /\left(1+\left(1-\omega_{0}\right)^{2 m}\right)
$$

after $m$ iterations. This compares with (2.13) and we again get results similar to (3.13) and (3.15).

In all cases above, we have considered a comparison of spectral radii. In actual computation, it is a matrix norm which determines the behavior of the iterative scheme. When the $B$ matrix has the form (2.7) and is real and symmetric, the spectral norm of all the above methods can be compared [5], [21]. (CCSOR is slightly the fastest.) In [22], other matrix norms are considered. In our case, the spectral norm appears somewhat difficult to obtain; however, some results on this are found in Section 8.4 of Young [21]. (Kincaid and Young have recently solved this problem.) Thus, comparing $S\left(\mathfrak{R}_{\omega_{0}}^{m}\right)$ with (3.19) seems to indicate that the SOR method is faster but the two are asymptotically equal. In actual practice, the SOR method is slightly slower but not apparently as much as in the real case (see Table I in Section 5).

4. The Unsymmetric Modified SOR (UNSOR). This method is defined by

$$
\begin{aligned}
u^{(n+1 / 2)} & =\omega_{1}\left(h^{2} L^{-1} v^{(n)}\right)+\left(1-\omega_{1}\right) u^{(n)}, \\
v^{(n+1 / 2)} & =\omega_{2}\left(-\frac{2}{h^{2}} L^{-1} M u^{(n+1 / 2)}+\frac{L^{-1} D}{h^{2}}\right)+\left(1-\omega_{2}\right) v^{(n)}, \\
v^{(n+1)} & =\omega_{3}\left(-\frac{2}{h^{2}} L^{-1} M u^{(n+1 / 2)}+\frac{L^{-1} D}{h^{2}}\right)+\left(1-\omega_{3}\right) v^{(n+1 / 2)}, \\
u^{(n+1)} & =\omega_{4}\left(h^{2} L^{-1} v^{(n+1)}\right)+\left(1-\omega_{4}\right) u^{(n+1 / 2)} .
\end{aligned}
$$

The method has been completely analyzed for matrices of the form (2.1) [20], [21]. The eigenvalues of the matrix of (4.1) are the same as those of

$$
R_{\omega_{1}+\omega_{0}-\omega_{1} \omega_{3}, \omega_{2}+\omega_{0}-\omega_{2} \omega_{0} \cdot}
$$

This has been analyzed in Section 2, where we have shown that the optimal $\omega$ 's are

$$
\omega_{1}+\omega_{3}-\omega_{1} \omega_{3}=\omega_{2}+\omega_{4}-\omega_{2} \omega_{4}=\omega_{0}=2 /\left(1+(1+2 \bar{\tau})^{1 / 2}\right),
$$

or

$$
\omega_{i}=1 \pm\left(1-\omega_{0}\right)^{1 / 2}, \quad i=1,2,3,4
$$


Since each iteration requires twice the amount of work of (2.2) and since the eigenvalues are the same, this approach promises nothing for the ordering of (2.1).

5. Results. Equation (2.2) can be written as
(a)

$$
L \tilde{u}=h^{2} v^{(n)},
$$

$$
u^{(n+1)}=\omega_{1} \tilde{u}+\left(1-\omega_{1}\right) u^{(n)},
$$

$$
\begin{gathered}
L \tilde{v}=-2 M u^{(n+1)} / h^{2}+D / h^{2}, \\
v^{(n+1)}=\omega_{2} \tilde{v}+\left(1-\omega_{2}\right) v^{(n)} .
\end{gathered}
$$

Now (5.1a) and (5.1c) require the solution of an $N^{2} \times N^{2}$ linear system. This in itself can be solved by SOR iteration. However, since the region is a square, and the form of the matrix so regular, we considered the method of Hockney [6], [7] and the fast Fourier transform. Modification of the method to fit our boundary conditions are described in [1], [2]. A comparison of the machine time required to solve (5.1a) by the method of Hockney with the time required for one SOR iteration indicates the system can be completely solved by Hockney's method in the same time as it takes for $2 \frac{1}{2}$ SOR iterations. Table I below indicates some machine results. These results were obtained at The Johns Hopkins Applied Physics Laboratory on the IBM 360/91 in PL1, with convergence criteria of

$$
\max _{i j}\left\{\left|u_{i j}^{(n+1)}-u_{i j}^{(n)}\right|,\left|v_{i j}^{(n+1)}-v_{i j}^{(n)}\right|\right\} \leqq 10^{-4}
$$

and

$$
u_{i j}^{(0)}=v_{i j}^{(0)}=0 .
$$

TABLE I. Mesh Size vs. Iteration

\begin{tabular}{rcc}
\multicolumn{4}{c}{$\bar{\tau}=.3 / h$} \\
\hline$h^{-1}$ & SOR & CCSOR \\
\hline 8 & 14 & 14 \\
16 & 20 & 19 \\
32 & 30 & 27 \\
64 & 42 & 40 \\
\hline
\end{tabular}

Applied Physics Laboratory

The Johns Hopkins University

Silver Spring, Maryland 20910

1. B. L. Buzbee, G. H. Golub \& C. W. Nielson, "On direct methods for solving Poisson's equations," SIAM J. Numer. Anal., v. 7, 1970, pp. 627-656.

2. R. COLONY \& R. R. REYNOLDS, An Application of Hockney's Method for Solving Poisson's Equation, Spring Joint Computer Conference (Atlantic City, N.J.), AFIPS Press, Montvale, N.J., 1970, pp. 409-416.

3. L. W. EHRLICH, "The block symmetric successive overrelaxation method," J. Soc. Indust. Appl. Math., v. 12, 1964, pp. 807-826. MR 33 \#5134.

4. L. W. EHRLICH, "Solving the biharmonic equation as coupled finite difference equations," SIAM J. Numer. Anal., v. 8, 1971, pp. 278-287. 
5. G. H. Golub \& R. S. VARGA, "Chebyshev semi-iterative methods, successive overrelaxation iterative methods, and second order Richardson iterative methods. I," Numer. Math., v. 3, 1961, pp. 147-156. MR 26 \#3207.

6. R. W. HockNeY, "A fast direct solution of Poisson's equation using Fourier analysis," J. Assoc. Comput. Mach., v. 12, 1965, pp. 95-113. MR 35 \#3913.

7. R. W. Hockney, "The potential calculation and some applications," in Methods in Computational Physics. Vol. 9, Academic Press, New York, 1970, pp. 135-211.

8. B. KREDELL, "On complex successive overrelaxation," Nordisk Tidskr. Informationsbehandling, v. 2, 1962, pp. 143-152. MR 29 \#1736.

9. W. Niethammer, "Relaxation bei komplexen Matrizen," Math. Z., v. 86, 1964, pp. 34-40. MR 29 \#6618.

10. W. Niethammer, "Úber-und Unterrelaxation bei linearen Gleichungssystemen," Computing, v. 5, 1970, pp. 303-311.

10a. W. NiETHAMMER, "Iterationsverfahren und allgemeine Euler-Verfahren," Math. Z., v. 102, 1967, pp. 288-317. MR 38 \#6741.

11. D. B. Russell, On Obtaining Solutions to the Navier-Stokes Equations with Automatic Digital Computers, Aeronautical Research Council, R\&M \#3331, Her Majesty's Stationery Office, London, 1963.

12. J. W. Sheldon, "On the spectral norms of several iterative processes," J. Assoc. Comput. Mach., v. 6, 1959, pp. 494-505. MR 23 \# B1647.

13. J. SMITH, "The coupled equation approach to the numerical solution of the biharmonic equation by finite differences. I," SIAM J. Numer. Anal., v. 5, 1968, pp. 323-339. MR 38 \#1847.

14. J. SMITH, "The coupled equation approach to the numerical solution of the biharmonic equation by finite differences. II,"SIAM J. Numer. Anal., v. 7, 1970, pp. 104-111. MR 42 \#7091.

15. R. S. VARGA, "A comparison of the successive overrelaxation method and semiiterative methods using Chebyshev polynomials," J. Soc. Indust. Appl. Math., v. 5, 1957, pp. 39-46. MR 19, 772.

16. R. S. VARGA, Matrix Iterative Analysis, Prentice-Hall, Englewood Cliffs, N.J., 1962. MR 28 \#1725.

17. H. E. WRIGLEY, "Accelerating the Jacobi method for solving simultaneous equations by Chebyshev extrapolation when the eigenvalues of the iteration matrix are complex," Comput. J., v. 6, 1963/64, pp. 169-176. MR 27 \#2095.

18. D. M. Young, "Iterative methods for solving partial differential equations of elliptic type,"Trans. Amer. Math. Soc., v. 76, 1954, pp. 92-111. MR 15, 562.

19. D. M. Young, "On Richardson's method for solving linear systems with positive definite matrices," J. Mathematical Phys., v. 32, 1954, pp. 243-255. MR 15, 650.

20. D. M. Young, "Convergence properties of the symmetric and unsymmetric sucessive overrelaxation methods and related methods," Math. Comp., v. 24, 1970, pp. 793-807.

21. D. M. Young, Iterative Solution of Large Linear Systems, Academic Press, New York, 1971 .

22. D. M. Young \& D. R. KIncaId, Norms of the Successive Overrelaxation Method and Related Methods, TNN-94, Computation Center, University of Texas, Austin, Tex., 1969.

23. D. M. Young, M. F. Wheeler \& J. A. Downing, On the Use of the Modified Successive Overrelaxation Method With Several Relaxation Factors, Proc. IFIP 65, W. A. Kalenich (Editor), Spartan Books, Washington, D.C., 1965, pp. 177-182. 